\title{
Theoretical Investigation of Transient Ultra-Thin Lubricant Film During Rapid Deceleration
}

\author{
M.F. Abd Al-Samieha \\ a Mechanical design \& production Department, Military Technical College, Cairo, Egypt.
}

Keywords:

Ultra-thin films

Transient Elastohydrodynamics

Solvation

Van der Waals' force

Electrostatic

\section{Corresponding author:}

M.F. Abd Al-Samieh

Mechanical design \& production

Department, Military Technical

College, Cairo, Egypt

E-mail:

mohamed.fahmy203@hotmail.com

\begin{abstract}
A B S T R A C T
A theoretical model using Newton-Raphson technique to study the behavior of ultra-thin film thickness formation subjected to transient speed conditions is presented in this paper. The transient oil film pressure and oil film thickness at various snap shots are shown by the numerical simulation. A comparison of the numerical results with experimental work of Glovnea and Spikes has been described and shows a good agreement for the case of rapid halting from a different speed. In the next stage, the formation of a lubricant film thickness in conjunctions of order of few nanometers has been discussed for the case where a polar lubricant is used as an intervening media between two mating molecularly smooth surfaces. The simulation study showed the significant effects of electrostatic force, Van der Waals' and solvation pressure on the lubrication film thickness formation under transient condition.
\end{abstract}

(C) 2018 Published by Faculty of Engineering

\section{INTRODUCTION}

Practical machine components undergo transient conditions during operations that can result in breakdown of the lubricant film thickness and as a consequence of that the life of these components can be reduced significantly. In real applications, lubricated contacts are subjected to variations of load, geometry or velocity of the contacting surfaces. For example, all these parameters vary during an operation cycle of gears and cams. Another example, in roller element bearings, the load changes when the rolling element enters the loading zone. Stepper motors subjected the bearings to sudden variations of speed in a repetitive startstop succession.
Several attempts were made to study the mechanism of transient elastohydrodynamic lubricated contact both theoretically and experimentally for the variation of applied load as shown by many researchers such as Wang et al. [1], Kalita and Kumar [2], Huang and Wang [3] and Zhang and Glovnea [4,5]. Glovnea and Spikes [6,7], Al-Samieh and Rahnejat [8], Petr et al. [9] and Ming et al. [10] and many other studied the transient film thickness variation due to surface velocity. The transient variation due to surface geometry is shown in many references such as shown in references [11-13]. The results of all the previous study both theoretically and experimentally showed that the film thickness observed in transient action is in contradiction with the predicted values using 
steady state elastohydrodynamic theory at instantaneous velocity as the film thickness will be zero as the entraining velocity falls to zero, which is in contradiction with the results of experimental transient work and also the results showed that the formation of a dimple-shaped film during the part of the cycle with the increasing squeeze effect.

Glovnea and Spikes [6,7] studied experimentally the transient elastohydrodynamic for the case of rapid halting, using ultrathin interferometry coupled to a high-speed camera to measure the change in lubricant film thickness, and theoretically based on Grubin's analytical solution for elastohydrodynamic lubrication. The results of their work show that the central film thickness at the end of halting depends little on the initial velocity but quite strongly depend on the rate of deceleration. Just before halting is finished, the film thickness in the inlet decreases rapidly to form a central entrapment.

Recent advances in technology have resulted in the downsizing of machine elements with very thin film conjunctions, typically in the order of or below $100 \mathrm{~nm}$. An example of this is the case of a hard disk drive system of a computer, where the flying height of a magnetic head over a disk surface can approach a few nanometers or microelectromechanical gears and actuators as shown by Al-Samieh [14]. These systems undergoing reciprocating motion or coming to a permanent halt. In such systems, it is usually assumed that, the film thickness can be predicted using steady state elastohydrodynamic theory but this is not the case, because as shown previously by many researchers such as studies of Jang and Tichy [15], Matsuoka and Kato [16] and Al-Samieh and Rahnejat [8] for ultra thin film, the film thickness formation is because of intermolecular force of solvation and Van der Waal's force in addition to the hydrodynamic action. In fact, all the previous mentioned study is for the non-polar lubricant acting as a separating media between two molecularly mica smooth surfaces. Recently, AlSamieh [17] studied the case for which a polar lubricant is drawn between two molecularly mica surfaces under steady state condition and his results showed that the effect of electrostatic double layer forces cannot be neglected in estimating the value of the film thickness under steady state condition.
In this paper a transient solution for the problem of ultrathin film considering hydrodynamic action, the ploraization of the lubricant, intermolecular force of solvation and Van der Waal's force can be studied using the iteration method of Newton-Raphson. A full numerical solution of the condition reported by Glovnea and Spikes [6,7] can be investigated under the condition of rapid halting. The methodology after that is extended to the case with the significant of the electrostatic force and structural and molecular interaction forces of Van der Waal's and solvation.

\section{BACKGROUND THEORY}

Matsuoka and Kato [16], Al-Samieh and Rahnejat [8] and Al-Samieh $[14,17,18]$ have shown that the load carried by the lubricant is supported by the pressure generation in the contacting region. Therefore, in the case of ultrathin film thickness for the situation where the intervening media is a polar lubricant, the total pressure can be given as:

$$
P=P_{s}+P_{v d w}+P_{e}+P_{h}
$$

\subsection{Transient elastohydrodynamic conjunction}

The Reynolds' equation determines the hydrodynamic pressure distribution for transient conditions and can be written in dimensionless form as:

$\frac{\partial}{\partial X}\left(\frac{\bar{\rho} H^{3}}{\bar{\eta}} \frac{\partial P_{h}}{\partial X}\right)+\frac{\partial}{\partial Y}\left(\frac{\bar{\rho} H^{3}}{\bar{\eta}} \frac{\partial P_{h}}{\partial Y}\right)=\lambda\left\{\frac{\partial(\bar{\rho} H)}{\partial X}+\frac{b E^{\prime}}{\eta_{o} u}\left(\bar{\rho} \frac{\partial H}{\partial \bar{t}}+H \frac{\partial \bar{\rho}}{\partial \bar{t}}\right)\right\}$

where the following dimensionless groups have been used:

$$
\begin{aligned}
& X=x / b, Y=y / b, \bar{\rho}=\rho / \rho_{o}, \bar{\eta}=\eta / \eta_{o}, H=h R / b^{2}, \\
& U=u \eta_{o} / R E^{\prime}, P_{h}=p_{h} / P_{h e r}, \bar{t}=E^{\prime} U t / \eta_{o}
\end{aligned}
$$

and: $\lambda=\frac{12 R^{2} \eta_{o} u}{b^{3} P_{h}}$

The film thickness in an elastohydrodynamic contact is described in dimensionless form as:

$$
H(t)=H_{o}(t)+S+\frac{R \delta(t)}{b^{2}}
$$

Where $\delta_{i, j}(\bar{t})$ is the total elastic deformation of the contiguous bodies in contact is given as shown by Al-Samieh and Rahnejat [8] The variation in density of the lubricant with 
pressure is described by the Dowson and Higginson model in 1959 as:

$$
\bar{\rho}=1+\frac{\varepsilon P_{h} P_{H e r}}{1+\xi P_{h} P_{H e r}}
$$

where: $\varepsilon$ and $\zeta$ are constants related to the type of lubricant employed.

The Roelands in 1966 presented equation expressing the influence of pressure upon viscosity has been adopted. In non-dimensional terms, it may be written in the form:

$$
\left.\bar{\eta}=\exp \left[\ln \eta_{\circ}+9.67\right]\left(1+5.1 * 10^{-9} P_{h} P_{H e r}\right)^{z}-1\right]
$$

where:

$$
\mathrm{Z}=\frac{\alpha}{5.1 * 10^{-9}\left[\ln \eta_{\circ}+9.67\right]}
$$

The Reynolds' equation can be solved using Newton-Raphson technique in the following numerical form:

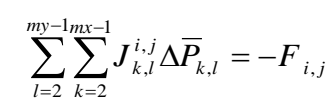

The details for the numerical solution of the Reynolds'equation is shown in more details by AlSamieh and Rahnejat [8] and Al-Samieh $[14,17,18]$.

\subsection{Molecular and Surface actions in narrow conjunctions}

The net force between two solids in a liquid include both the Van der Waals and electrostatic double-layer force and at small surface separations the attractive Van der Waals and repulsive electrostatic forces often fail to describe their interaction and the effect of molecular force of solvation is dominant in determining the oil film thickness.

Many researchers (such as Canova [19], Christensen and Horn [20], Thaicharoen and Schwarzkopf [21], Prakash [22], Morciano et al. [23] and Peiyuan et al. [24]) have studied both experimentally and theoretically the behaviour of the above mentioned forces of Van der Waals, electrostatic double-layer force and solvation. Israelachvili [25] gives the pressure in the fluid, induced by the Van der Waals' forces, solvation and electrostatic double layer force as a function of distance as follows:

$$
P_{v d w}=\frac{-A}{6 \pi h^{3}}
$$

$$
\begin{aligned}
& P_{s}=-C e^{\frac{-h}{a}} \cos (2 \pi h / a) \\
& p_{\text {elest }}=2 \varepsilon \varepsilon_{o} \kappa^{2} \omega_{0}{ }^{2} e^{-\kappa h}
\end{aligned}
$$

where, $\varepsilon$ is the dielectric constant of the liquid (for propylene carbonate=65), $\varepsilon_{o}$ is the dielectric permittivity of the free space (8.854X10-12 (farads/meter), $k$ is the Debye length and $\omega_{0}$ is the surface potential.

The details of the above mentioned forces are shown in detail by Al-Samieh and Rahnejat [8] and Al-Samieh $[14,17,18]$.

\section{RESULTS AND DISCUSSION}

Simulations studies are carried out to show transient condition of rapid halting on the formation of film thickness and pressure distribution. The experimental work done by Glovnea and Spikes [6] has been compared with the results of the current analysis. They measured lubricant film behaviour using ultrathin film interferometry. The applied contact load of $20 \mathrm{~N}$, in all experiments was used at a controlled lubricant temperature of $50{ }^{\circ} \mathrm{C}$. The properties of oil and materials used in their experiments are given in Table 1 . Figure 1 shows the entraining speed variation with time that can be imposed for all the conditions described in this paper.

Table 1. Lubricant and material Properties used by Glovnea and Spikes [6].

\begin{tabular}{|c|c|c|c|}
\hline Viscosity $\eta_{\mathbf{o}}$ & $\begin{array}{c}0.609 \\
\text { Pa.s }\end{array}$ & $\begin{array}{c}\text { Young's } \\
\text { modulus, } \mathbf{E}_{\mathbf{A}}\end{array}$ & $\begin{array}{c}0.746 \times 10^{11} \\
\mathrm{~Pa}\end{array}$ \\
\hline $\begin{array}{c}\text { Pressure of } \\
\text { viscosity } \\
\text { coefficient } \boldsymbol{\alpha}\end{array}$ & $\begin{array}{c}24 \mathrm{X} 10^{-9} \\
\mathrm{~Pa}^{-1}\end{array}$ & $\begin{array}{c}\text { Young's } \\
\text { modulus, } \mathrm{E}_{\mathrm{B}}\end{array}$ & $\begin{array}{c}0.211 \mathrm{X} 10^{12} \\
\mathrm{~Pa}\end{array}$ \\
\hline $\boldsymbol{\varepsilon}$ & $5.83 \times 10^{-10} \mathrm{~Pa}$ & $\begin{array}{c}\text { Poisson's } \\
\text { ratio } \boldsymbol{v}_{\boldsymbol{A}}\end{array}$ & 0.25 \\
\hline$\xi$ & $1.68 \times 10^{-9} \mathrm{~Pa}$ & $\begin{array}{c}\text { Poisson's } \\
\text { ratio } \boldsymbol{v}_{\mathbf{B}}\end{array}$ & 0.3 \\
\hline $\begin{array}{c}\text { Radius of } \\
\text { ball } \boldsymbol{R}\end{array}$ & $0.01 \mathrm{~m}$ & & \\
\hline
\end{tabular}

Four conditions, as in the case of the experimental work of Glovnea and Spikes [6] depending on the initial speed of entraining motion, have been investigated for purpose of comparison. These initial speeds are $0.5,0.3,0.2$ and $0.1 \mathrm{~m} / \mathrm{s}$ respectively. 


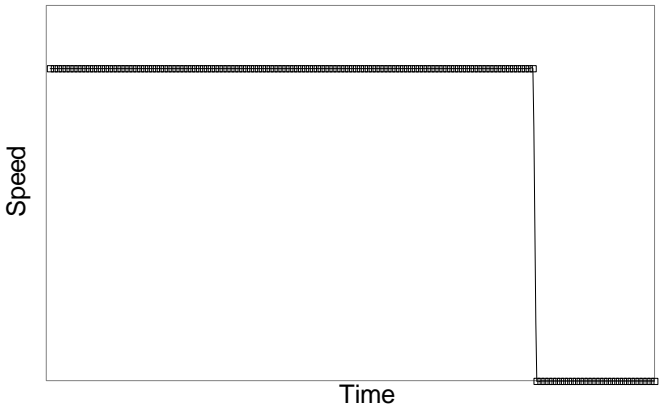

Fig. 1. Speed profile with time variation.

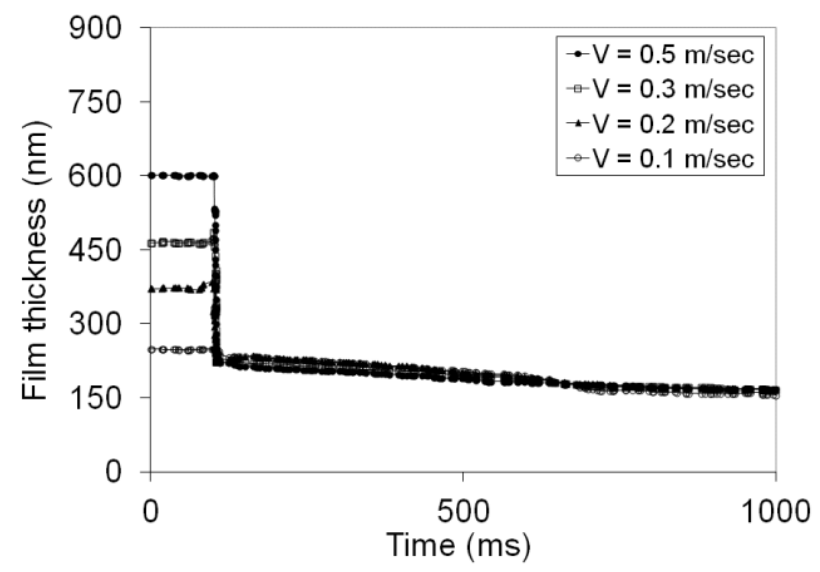

Fig. 2. Experimentally measured central film thickness against time for different initial speed after Glovnea and Spikes [6].

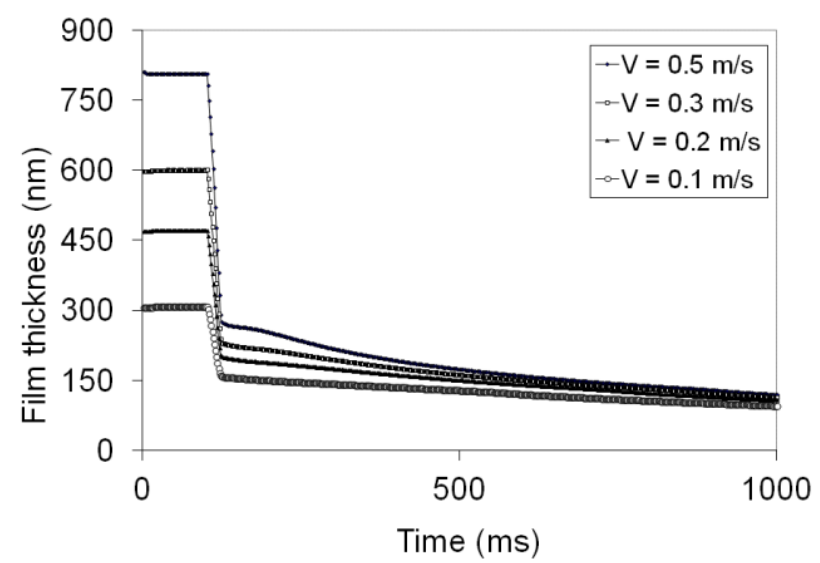

Fig. 3. Numerically predicted central film thickness against time for different initial speed.

Figure 2 shows the central oil film thickness variation with time during halting from a different speed that expected from the experimental work of Glovnea and Spikes [6]. Figure 3 shows the same behaviour that obtained from the current numerical solution under the same condition where the hydrodynamic action is the only force considered in calculating the film thickness and pressure distribution. Very good agreement is clear by comparisons of the numerical solutions with the experimental works of Glovnea and Spikes [6]. This agreement is qualitative because the piezo-viscosity index for polyalphaolefin (PAO) lubricant is different as given by Glovnea and Spikes [6] at different oil temperature indicate that the PAO behaves as non-Newtonian lubricant, whereas the current analysis is for the Newtonian case. The same conclusion was found by Al-Samieh and Rahnejat [8] for studying the transient ultra-thin film where PFPEZ lubricant was used. However, in both figures, it is obvious that, the film thickness collapse in two stages. In the first stages, the film thickness falls very rapidly (almost instantly) to a certain value. In the second stage, the film thickness gradually decreases because of pure squeeze action which has also been observed by Petr et al. [9] and Ming et al. [10].

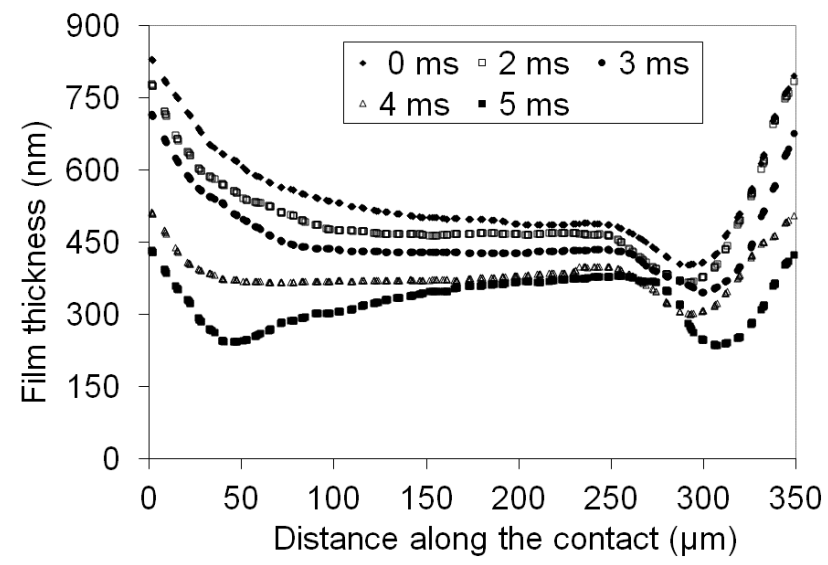

Fig. 4. Experimentally film thickness profile along the contact for initial speed $0.3 \mathrm{~m} / \mathrm{s}$ after Glovnea and Spikes [6].

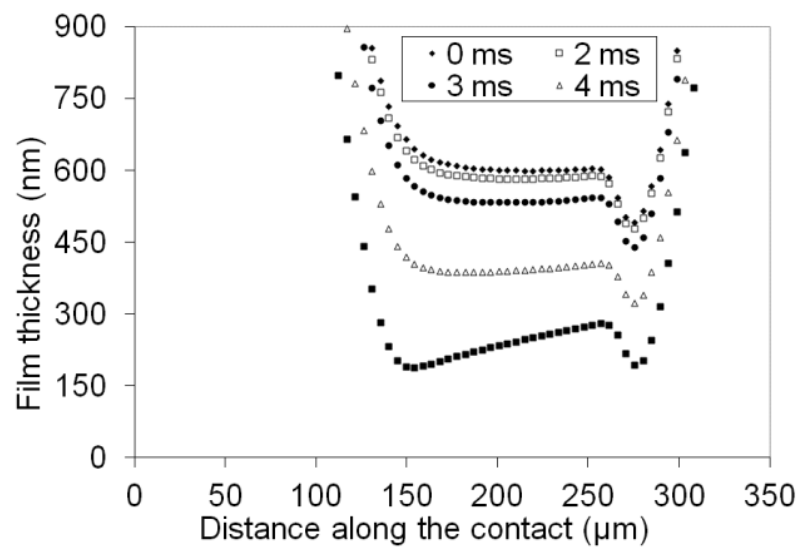

Fig. 5. Numerically predicted film thickness profile along the contact for initial speed $0.3 \mathrm{~m} / \mathrm{s}$.

Figures 4 and 5 show the elastic film shapes along the contact for the experimental results of Glovnea and Spikes [6] and that numerically predicted for the previous condition during sudden halting from an initial speed of $0.3 \mathrm{~m} / \mathrm{s}$ respectively. As can be 
seen, good agreement between both sets of results. In the first stage (corresponding to $0 \mathrm{sec}$ that shown in Figs. 4 and 5), the shape of the film thickness resembles the shape of the elastohydrodynamic film in that a restriction in the film thickness profile is appeared. During the second stage after the cessation of entraining motion (corresponding to 2, 3, 4 and $5 \mathrm{~ms}$ that shown in Figs. 4 and 5), the central film thickness show gradual reduction due to the stopping of entraining motion and therefore, an insufficient amount of lubricant is drawn into the contact because of the cessation of the entraining motion. The experimental work shown by Glovnea and Spikes [6] and the numerical study showed that an entrapped lubricant film shown in both Figs. 4 and 5 (corresponding to 4 and $5 \mathrm{~ms}$ ) cause the film thickness to be reduced gradually during the period of cessation of motion. After a long enough period of cessation of entraining motion, the central film thickness gradually diminishes results that the two surfaces stick together.

In the results described above the numerical predicted value of central lubricant film thickness is never below $100 \mathrm{~nm}$, and the media used as a lubricant of polyalphaolefin (PAO) is non-polar lubricant, so, the action from the action of electrostatic force, solvation and Van der Waals force is not appear. To show their contributions in determining the oil film thickness, the material and lubricant properties given in Table 2 are used in the simulation study together with transient conditions for the case study of rapid halting from a certain speed. The load and speed of entraining motion are chosen where the action of electrostatic force and the intermolecular force has a significant effect on the formation of the film thickness. In the simulation study an initial speed of $0.1 \mathrm{~mm} / \mathrm{s}$ and an applied load of $25 \mathrm{mN}$ are used.

Table 2. Physical and geometrical properties of contacting materials and PC lubricant.

\begin{tabular}{|l|c|l|c|}
\hline Viscosity $\eta_{\mathrm{o}}$ & $2.8 \mathrm{mPa} . \mathrm{s}$ & $\begin{array}{l}\text { Pressure of } \\
\text { viscosity } \\
\text { coefficient } \boldsymbol{\alpha}\end{array}$ & $10 \mathrm{GPa}^{-1}$ \\
\hline $\boldsymbol{\varepsilon}$ & $5.83 \times 10-10 \mathrm{~Pa}$ & Poisson's ratio $\boldsymbol{v}_{\boldsymbol{A}}$ & 0.205 \\
\hline $\begin{array}{l}\text { Molecular } \\
\text { diameter, } \boldsymbol{a}\end{array}$ & $0.5 \mathrm{~nm}$ & Poisson's ratio $\boldsymbol{v}_{\mathrm{B}}$ & 0.205 \\
\hline $\begin{array}{l}\text { Hamaker } \\
\text { constant, } \boldsymbol{A}\end{array}$ & $\begin{array}{l}5.51 \mathrm{X} 10^{-20} \\
\text { Joules }\end{array}$ & $\begin{array}{l}\text { Young's } \\
\text { modulus } \boldsymbol{E}_{\mathrm{A}}\end{array}$ & $34.5 \mathrm{GPa}$ \\
\hline $\begin{array}{l}\text { Constant } \\
\text { defined in } \\
\text { eq. }(8), \boldsymbol{C}\end{array}$ & $172 \mathrm{MPa}$ & $\begin{array}{l}\text { Young's } \\
\text { modulus } \boldsymbol{E}_{\boldsymbol{B}}\end{array}$ & $34.5 \mathrm{GPa}$ \\
\hline $\boldsymbol{\xi}$ & $1.68 \times 10^{-9} \mathrm{~Pa}$ & Radius of ball $\boldsymbol{R}$ & $0.01 \mathrm{~m}$ \\
\hline
\end{tabular}



Fig. 6. Numerically predicted central film thickness against time as the result of viscous action.

Figure 6 shows the transient oil film thickness in the central of contact during a rapid halting from an initial entraining motion of $0.1 \mathrm{~mm} / \mathrm{sec}$ for inactive period of motion of $2 \mathrm{sec}$ for the case where the hydrodynamic action is the only force considered in the simulation study to predict the film thickness and pressure distribution for ultrathin film, under the operating conditions discussed before. The inactive period of motion in the simulation study was chosen as $2 \mathrm{sec}$, in such that, by increasing the period of inactive motion greater than $2 \mathrm{sec}$, the minimum film thickness results show a negative value indicating that no surface film separating the two adjacent surfaces. It is clear that, from figure 6, the film thickness collapses in two stages as mentioned before. In the start of motion, the value of the film thickness at the centre of contact is $18 \mathrm{~nm}$ as the numerical solutions predicted. The regime of lubrication in this stage belongs to iso-viscous rigid regime in the Greenwood chart. The corresponding calculated values according to Brewe el al. [26] central film thickness formula is $18.16 \mathrm{~nm}$. The error is being less than $1 \%$. When the entraining motion is halted, the central film thickness has rapidly collapsed to a value of $2.5 \mathrm{~nm}$, and from which the central film thickness decays subsequently as expected due to pure squeeze action reaches a value of $0.5 \mathrm{~nm}$ at the end of the motion. By further increasing the period of inactive motion, the film thickness value tends to zero and the two contacting surfaces would come together and stick to each other resulting in more wear and damage of the operating element. In fact, the central film thickness shown in Fig. 6 is not justified, as it abrogates the rigidity of the Newtonian hard molecular spheres, because the film thickness reaches a value less than 2 molecular diameters of the intermediate fluid 
(the film thickness reaches a value of $0.5 \mathrm{~nm}$ ) as discussed previously by Al-Samieh and Rahnejat [8]. However, this is not the case, because other forces are important when calculating the oil film thickness in such narrow gaps. These forces are the effects of Van der Waals' and the electrostatic forces, and as the film thickness is reduced further, the solvation forces must be included, as shown by Matsuoka and Kato [16], Al-Samieh and Rahnejat [8] and Al-Samieh [14,17,18] and this case is the subject of the next part of the paper.

Figure 7 shows the transient central oil film thickness during a rapid halting from an initial speed of entraining motion of $0.1 \mathrm{~mm} / \mathrm{sec}$ for different period of inactive motion ranging from $2 \mathrm{sec}$ to $9 \mathrm{sec}$, for the case where the electrostatic force and the intermolecular force of solvation and Van der Waal's force and the hydrodynamic action is considered in calculation of the predicted value of film thickness. As shown in Fig. 7, the same oil film thickness characteristics were observed as in case shown in Fig. 6 in that the film thickness collapse in two stages. In the start of motion, the film thickness has the value of $24 \mathrm{~nm}$ in comparison to that shown in Fig. 6 of $18 \mathrm{~nm}$, the only difference is the increase the value of film thickness. It is obvious that, the film thickness is increased to about $33 \%$. This increased value of the film thickness is due to the action of the electrostatic force as shown previously by AlSamieh [17]. He showed that for separations more than five molecular diameters of the intervening polar liquid, the formation of a lubricant film thickness is governed by combined effects of hydrodynamic action and intermolecular force of a Van der Waals force and a double layer force. During the second stage, in the regions of inactive motion, the initial film thickness rapidly dropped to a value of $2.5 \mathrm{~nm}$. The film thickness shown in Fig. 7 differ that shown in figure 6 in that the film thickness values remains constant throughout the contact region. As the period of inactive motion is increased, the central film thickness values remains constant indicating that a unique value of the oil film thickness exists. This unique value because of solvation effect as shown previously by Matsuoka and Kato [16], AlSamieh and Rahnejat [8] and Al-Samieh $[14,17,18]$. Therefore, the transient ultrathin film thickness formation in this case is formed as a combined effect of the electrostatic force, intermolecular force of Van der Waal's and solvation and the hydrodynamic action and depends on the value of the film thickness.

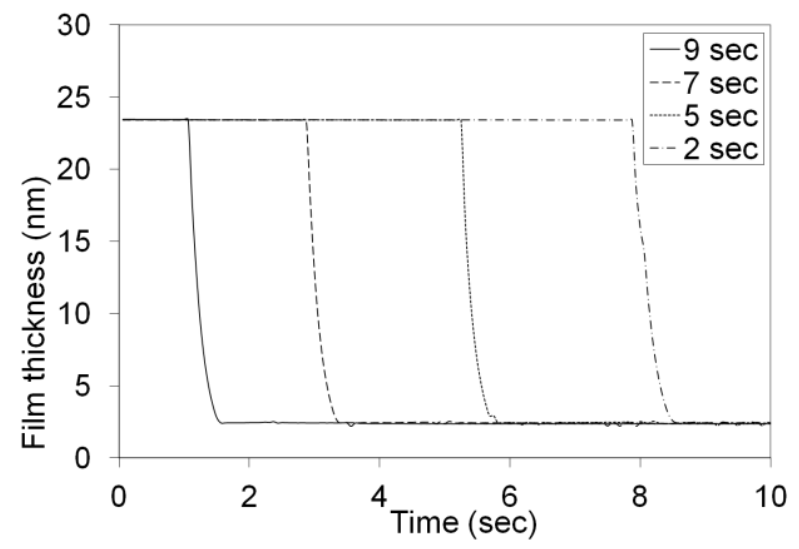

Fig. 7. Numerically predicted central film thickness against time as the result of combined viscous action and Van der Waals and the electrostatic double layer forces for different period of inactive motion.

The oil film thickness shape and the corresponding pressure distribution at certain snap shots of time for the case where the period of inactive motion is $9 \mathrm{sec}$ are shown in Fig. 8. It is clear that from the shape of the pressure distribution that shown in Fig. 8a, at the start of motion, at $0.75 \mathrm{sec}$ (corresponding to $0.25 \mathrm{sec}$ before stopping) the dominant effect of hydrodynamic action and the electrostatic force in determining the transient oil film thickness. After cessation of entraining motion, the transient solvation pressure is aided to the hydrodynamic shape, this is clear as shown in Fig. 8b. The film thickness value is about $6 \mathrm{~nm}$. As the time of inactive motion increases as the value of the film thickness decrease to a value of $2.5 \mathrm{~nm}$. This is shown in Fig. 8c after $1 \mathrm{sec}$ from ceasing the motion, where from the shape of the pressure distribution, the oscillatory nature of solvation is aided to the hydrodynamic shape, therefore, the oil film thickness formation is mainly due to the action of solvation pressure. Referring back, to Fig. 8c, the flattened shape of the film thickness instead of the dimple shape which is the characteristic of the pure squeeze action indicate that the increased in the film thickness is due to the effect of the intermolecular force of solvation. This figure also shows the insignificant contribution due to the viscous effect. Figures $8 \mathrm{~d}$ and $8 \mathrm{e}$ shows that the pressure distribution and film thickness shape, after 5 sec from ceasing of motion and at the end of stopping motion i.e. after $9 \mathrm{sec}$ from ceasing of motion respectively. The 
pressure distribution and film thickness shape sown in Fig. 8d is similar to that shown in Figs. 8e and 8c. These figures also illustrate the insignificant contribution due to the viscous effect. The film thickness remains constant throughout this period. Therefore, under the aforementioned conditions, the film thickness formation in transient ultrathin film is formed as results of different theories including the hydrodynamic theory, continuum theories for the attractive Van der Waals force and the PoissonBoltzmann equation for the repulsive double layer electrostatic force and at small separation solvation pressure theory.

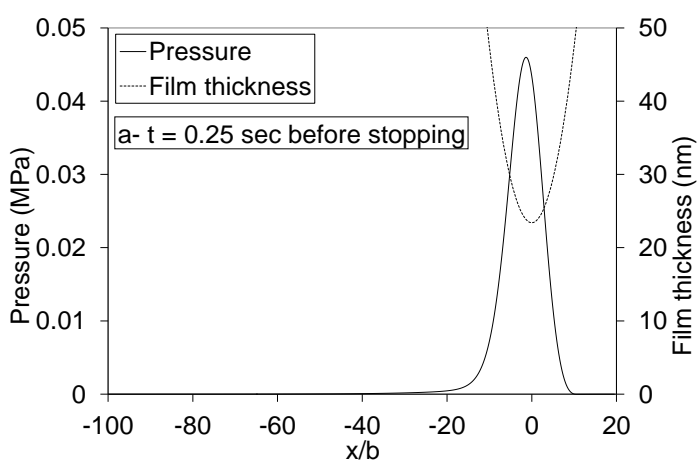

a)

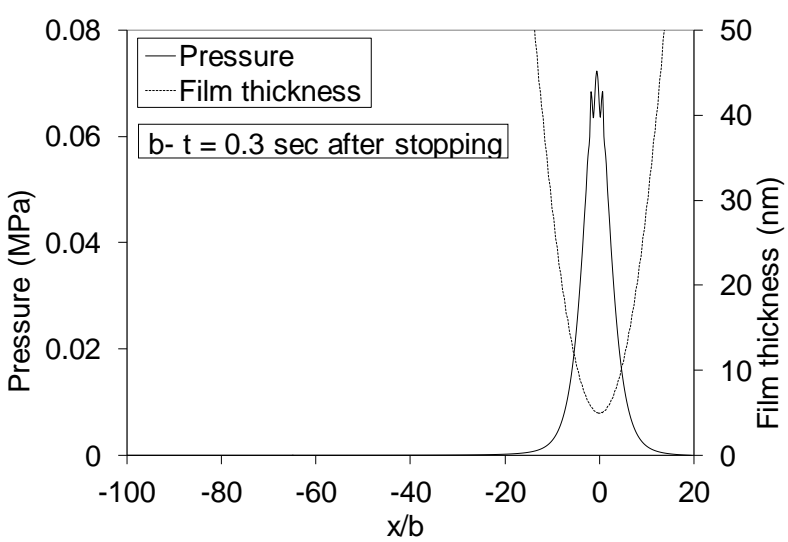

b)

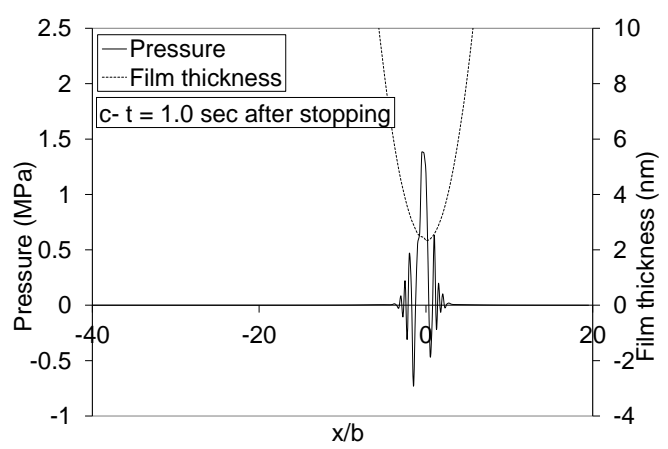

c)



d)

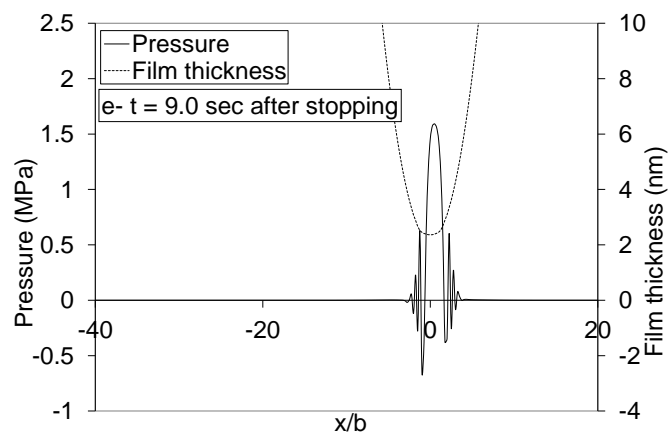

e)

Fig. 8. Pressure profile and film shape in the central line of contact as the result of combined viscous action and Van der Waals and the electrostatic double layer forces at different snap shots.

Unfortunately, there is no experimental or previous numerical work in the field of transient ultra-thin film thickness lubrication formation under the miscellaneous theories due to effect of electrostatic double layer force and the intermolecular force of solvation pressure and Van der Waal's force to compare with the second part of results in this paper.

\section{CONCLUSION}

The predictions of the mathematical model using Newton-Raphson technique have been compared to the measured film thickness data in the central of contact published by Glovnea and Spikes [6]. The results show a qualitative agreement because the intervening media of PAO behaves as nonNewtonian lubricant whereas the current analysis is for the Newtonian case. This agreement has open the way to study the transient ultra-thin film formation under the action of double layer electrostatic force due to polarization of the 
intermrdiate media and the Van der Waals' force (i.e. the action of DLVO theory after Derjaguin, Landau, Verwey, and Overbeek) and the effects of intermolecular force of solvation. The results show that the film thickness formation in a narrow gap is formed as a result of many theories. For the case study of start-stop discussed in this paper, at the begging of motion, the film thickness is formed due to combined effects of hydrodynamic action and the effects of double layer electrostatic force and the Van der Waals' force. As the entraining motion is halted, theoretically as predicted by the classical hydrodynamic theory the film thickness tends to zero and because of that the two mating surfaces stick to each other resulting in more wear and cause damage of the mechanical parts. Fortunately, this is not the situation, as shown in the result section; the film thickness after the motion is halted rapidly falls to a certain value and remains constant indicate that a unique value of the film thickness and this unique value are independent of the period of inactive motion. This unique value of film thickness as the action of the molecular force of solvation.

\section{REFERENCES}

[1] Y.Q. Wang, Z. He, W. Su, Effect of Impact Load on Transient Elastohydrodynamic Lubrication of Involute Spur Gears, Tribology Transactions, vol. 55 , iss. 2, pp. 155-162, 2012, doi: 10.1080/10402004.2011.639048

[2] T.J. Kalita, P. Kumar, Effect of Load Variation on Elastohydrodynamic Lubrication Film Collapse, Applied Mechanics and Materials, vol. 592-594, pp. 1366-1370, 2014, doi: 10.4028/www.scientific.net/AMM.592-594.1366

[3] X. Huang, Y. Wang, Transient Elastohydrodynamic Lubrication Analysis of Spur Gears under Oscillation Conditions, Lubrication Engineering, vol. 40, iss. 11, pp. 100-109, 2015.

[4] X. Zhang, R.P. Glovnea, The behaviour of lubricated EHD contacts subjected to vibrations, IOP Conference Series: Materials Science and Engineering, vol. 174, pp. 1-8, 2017, doi: 10.1088/1757-899X/174/1/012036

[5] X. Zhang, R.P. Glovnea, EHD Film Thickness Measurement in a Ball Bearing by Electrical Capacitance, in $70^{\text {th }}$ Annual Meeting and Exhibition of Society Tribologists and Lubrication Engineers, 17-21 May, 2015, Dallas, Texas, USA.

[6] R.P. Glovnea, H.A. Spikes, Elastohydrodynamic film collapse during rapid deceleration, Part I: experimental results, Journal of Tribology, vol. 123, iss. 2, pp. 254-261, 2001, doi: 10.1115/1.1308011

[7] R.P. Glovnea, H.A. Spikes, Elastohydrodynamic film collapse during rapid deceleration, Part II: theoretical analysis and comparison of theory to experiment, Journal of Tribology, vol. 123, iss. 2, pp. 262-267, 2001, doi: 10.1115/1.1308012

[8] M.F. Al-Samieh, H. Rahnejat, Ultra-thin lubricating films under transient conditions, J Journal of Physics D: Applied Physics, vol. 34, no. 17, pp. 2610-2621, 2001, doi: 10.1088/0022-3727/34/17/307

[9] S. Petr, K. David, K. Ivan, H. Martin, Experimental investigation of lubrication film formation at start-up of smooth surfaces, Science Journal, vol. 12, no. 1, pp. 825-828, 2015, doi: 10.17973/MMSJ.2015_12_201529

[10] C.L. Ming, Y.P. Chang, J. Sheu, Elastohydrodynamic lubrication of circular contacts at pure squeeze motion involving the mixture of two lubricants, Journal of Marine Science and Technology, vol. 23, no. 4, pp. 516-522, 2015, doi: 10.6119/JMST-0150325-1

[11] X. Huang, Q. Liu, Y. Wang, N. Dong, Transient Elastohydrodynamic Lubrication Analysis of Spur Gear Running-in Considering Effects of Solid Particles, Proceedings of the 2015 International Conference on Power Electronics and Energy Engineering, pp. 74-78, 2015, doi: 10.2991/peee15.2015 .20

[12] X. Huang, Y. Wang, Transient elastohydrodynamic lubrication analysis of spur gears running-in considering effects of solid particles and surface roughness, Industrial Lubrication and Tribology, vol. 68, iss. 2, pp. 183-190, 2016, doi: 10.1108/ILT-05-2015-0061

[13] F. Ildiko, S. Petr, H. Martin, Transient calculations in elastohydrodynamically lubricated point contacts, Engineering Mechanics, vol. 21, no. 5, pp. 311-319, 2014.

[14] M.F. Al-Samieh, Effect of Changing Ellipiticity Ratio on the Formation of Ultra-Thin Lubricating Film, Tribology in Industry, vol. 39, no. 4, pp. 431-443, 2017, doi: 10.24874/ti.2017.39.04.02

[15] S. Jang, J. Tichy, Rheological models for thin film EHL contacts, Journal of Tribology, vol. 117, iss. 1, pp. 22-28, 1995, doi: 10.1115/1.2830602

[16] H. Matsuoka, T. Kato, An ultrathin liquid film lubrication theory-Calculation method of solvation pressure and its application to the EHL problem, Journal of Tribology, vol. 119, iss. 1, pp. 217-226, 1997, doi: 10.1115/1.2832464

[17] M.F. Al-Samieh, Film thickness formation in nanoscale due to effects of Elastohydrodynamic, Electrostatic and Surface force of Solvation and Van 
der Waals, Tribology in industry, vol. 39, no. 1, pp. 120-128, 2017, doi: 10.24874/ti.2017.39.01.13

[18] M.F. Al-Samieh, Demarcation boundary between region dominated by elastohydrodynamic lubrication and that by the surface force action for different elliptical ratios, Industrial Lubrication and Tribology, vol. 69, iss. 2, pp. 215-224, 2017, doi: 10.1108/ILT-01-2016-0003

[19] G.H.L. Canova, A.J. Thomas, M. Ernst, Van der Waals interactions and the limits of isolated atom models at interfaces, Nature Communications, vol. 7, pp. 1-7, 2016, doi: 10.1038/ncomms11559

[20] H.K. Christensen, R.G. Horn, Direct measurement of the force between solid surfaces in a polar liquid, Journal of Chemical physical letters, vol. 98, iss. 1, pp. 45-48, 1983, doi: 10.1016/00092614(83)80199-7

[21] N. Thaicharoen, A. Schwarzkopf, G. Raithel, Measurement of van-der-Waals interaction by atom trajectory imaging, Physical Review A, vol. 92, iss. 4, pp. 1-6, 2015, doi: 10.1103/PhysRevA.92.040701

\section{NOMENCLATURE}

a : Lubricant molecular diameter

A : Hamaker constant

b : Radius of Hertzian contact region

C : Constant defined in equation (8) $=172 \mathrm{MPa}$

D : Deformation influence coefficient matrix

$\mathrm{E}_{\mathrm{A}, \mathrm{B}} \quad$ : Young's modulus of elasticity

E' : Reduced modulus of elasticity

$\mathrm{G}^{*} \quad$ : Materials' parameter, $\quad \mathrm{G}^{*}=\mathrm{E}^{\prime} \alpha$

h : Lubricant film thickness

H : Dimensionless film thickness, $\mathrm{H}=\mathrm{hR} / \mathrm{b} 2$

$\mathrm{H}_{0} \quad$ : Dimensionless central oil film thickness

l : Dimensionless side leakage boundary distance

$\mathrm{m} \quad$ : Dimensionless inlet distance

$\mathrm{n}_{\mathrm{x}}, \mathrm{n}_{\mathrm{y}} \quad$ : Number of computational grid nodes

P : Total contact pressure

ph : Hydrodynamic pressure

$\mathrm{p}_{\mathrm{s}} \quad$ : Solvation pressure due to surfaces' interaction

Pe : Electrostatic pressure

$\mathrm{p}_{\mathrm{vdw}} \quad$ : Pressure due to molecular Van der Waals' force

$\mathrm{P} \quad$ : Dimensionless total contact pressure, $\mathrm{P}=\mathrm{p} / \mathrm{PHer}$

$\mathrm{P}_{\mathrm{h}} \quad$ : Dimensionless hydrodynamic pressure, $\mathrm{P}_{\mathrm{h}}=\mathrm{p}_{\mathrm{h}} / \mathrm{P}_{\text {Her }}$

$P_{H e r} \quad$ : Maximum Hertzian contact pressure

$\mathrm{P}_{\mathrm{s}} \quad$ : Dimensionless solvation pressure, $\mathrm{P}_{\mathrm{s}}=\mathrm{p}_{\mathrm{s}} / \mathrm{P}_{\text {Her }}$

$\mathrm{Pe}_{\mathrm{e}} \quad$ : Dimensionless electrostatic pressure, $\mathrm{P}_{\mathrm{e}}=\mathrm{p}_{\mathrm{e}} / \mathrm{P}_{\mathrm{Her}}$

$\mathrm{P}_{\mathrm{vdw}} \quad$ : Dimensionless Van der Waals' pressure, $\mathrm{P}_{\mathrm{vdw}}=\mathrm{p}_{\mathrm{vdw}} / \mathrm{P}_{\mathrm{Her}}$

$\mathrm{N} \quad$ : Total number of mesh points

$\mathrm{K} \quad$ : Elliptical ratio

$\mathrm{R} \quad$ : Reduced radius of counterformal contact

w : Normal applied contact load
[22] C.M. Prakash, Effect of Surface Forces on Ultrathin Film Lubrication, ISRN Tribology, vol. 2014, no. 1, pp. 1-9, 2014, doi: 10.1155/2014/612195

[23] M. Morciano, M. Fasano, A. Nold, C.C. Braga, P. Yatsyshin, D.N. Sibley, B.D. Goddard, E. Chiavazzo, P. Asinari, S. Kalliadasis, Nonequilibrium molecular dynamics simulations of nanoconfined fluids at solid-liquid interfaces, Journal of Chemical Physics, vol. 146, no. 24, pp. 1-13, 2017, doi: 10.1063/1.4986904

[24] S. Peiyuan, Z. Meirong, J. Jile, Z. Yelong, H. Yaqian, S. Le, The Differential Method for Force Measurement Based on Electrostatic Force, Journal of Sensors, vol. 2017, pp. 1-7, 2017, doi: $10.1155 / 2017 / 1857920$

[25] J.N. Israelachvili, Intermolecular and surface forces. New York, Acdemaic Press, 2010.

[26] D. E. Brewe, B.J. Hamrock, C.M. Taylor, Effect of geometry on hydrodynamic film thickness, Journal of Tribology, vol. 101, iss. 2, pp. 231-239, 1979, doi:10.1115/1.3453332

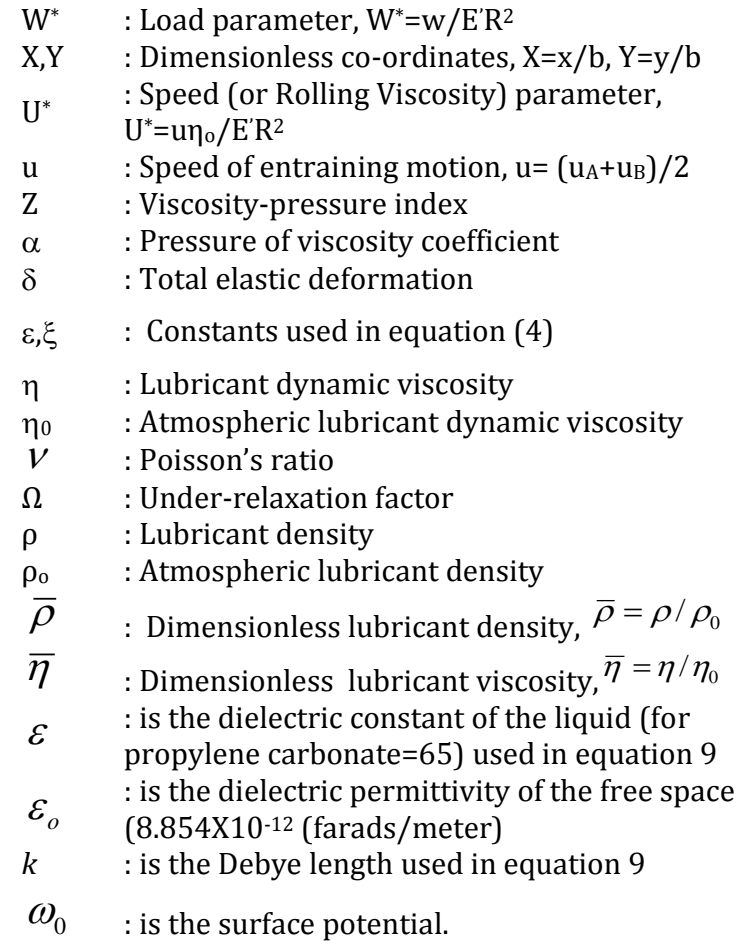

Superscripts:

$\mathrm{i}, \mathrm{j} \quad$ : Contravariant influence coefficient indices

$\mathrm{n} \quad$ : Iteration index

Subscripts:

A,B : Denote the contiguous bodies in contact

$\mathrm{k}, \mathrm{l} \quad$ : Covariant influence coefficient indices 